\title{
Anti-CD47 Monoclonal Antibody SHR-1603
}

National Cancer Institute

\section{Source}

National Cancer Institute. Anti-CD47 Monoclonal Antibody SHR-1603. NCI Thesaurus. Code C160202.

A monoclonal antibody targeting the human cell surface antigen CD47, with potential phagocytosis-inducing and antineoplastic activities. Upon administration, anti-CD47 monoclonal antibody SHR-1603 preferentially binds to CD47 on tumor cells. This blocks the interaction of CD47 with signal regulatory protein alpha (SIRPalpha), an inhibitory protein expressed on macrophages and dendritic cells (DCs), which prevents CD47/SIRPalpha-mediated signaling and abrogates the CD47/SIRPalpha-mediated inhibition of phagocytosis. This induces pro-phagocytic signaling mediated by the binding of calreticulin (CRT), which is specifically expressed on the surface of tumor cells, to lowdensity lipoprotein (LDL) receptor-related protein (LRP), expressed on macrophages, which results in macrophage activation and the specific phagocytosis of tumor cells. Additionally, blocking CD47 signaling activates both an anti-tumor T-lymphocyte immune response and T-cell-mediated killing of CD47-expressing tumor cells. CD47, also called integrin-associated protein (IAP), is a tumor-associated antigen (TAA) expressed on normal, healthy hematopoietic stem cells (HSCS) and overexpressed on the surface of a variety of cancer cells. Expression of CD47, and its interaction with SIRPalpha, leads to the inhibition of macrophage activation and protects cancer cells from phagocytosis, which allows cancer cells to proliferate. 